Published in final edited form as:

Vaccine. 2009 November 16; 27(49): 6840-6844. doi:10.1016/j.vaccine.2009.09.016.

\title{
How much will it hurt? HPV vaccine side effects and influence on completion of the three-dose regimen
}

\author{
Paul L. Reiter ${ }^{a, b,{ }^{*},}$ Noel T. Brewer ${ }^{a, b}$, Sami L. Gottlieb ${ }^{c}$, Annie-Laurie McRee ${ }^{a}$, and Jennifer \\ S. Smith ${ }^{a, b}$ \\ aUNC Gillings School of Global Public Health, United States \\ bLineberger Comprehensive Cancer Center, United States \\ ${ }^{\circ}$ Centers for Disease Control and Prevention, United States
}

\begin{abstract}
We examined the prevalence of reported pain following human papillomavirus (HPV) vaccination and whether it differed from that for other adolescent vaccines or affected completion of the HPV vaccine regimen. In 2008, we conducted cross-sectional surveys with parents of adolescent girls aged 11-20 living in areas of North Carolina with elevated cervical cancer rates who had received at least one dose of HPV vaccine. Pain from HPV vaccination, while commonly reported by parents, was less frequent compared to other adolescent vaccines and did not appear to affect vaccine regimen completion. These findings may be important to increase HPV vaccination coverage.
\end{abstract}

\section{Keywords \\ HPV; Vaccine; Side effects}

\section{Introduction}

Human papillomavirus (HPV) vaccine uptake remains modest among eligible females in the US. In recent large studies, only about $25 \%$ of adolescent girls had initiated HPV vaccine by early 2008, with even fewer having completed the full three-dose regimen [1,2]. Parents are less likely to get HPV vaccine for their adolescent daughters if they are concerned about adverse events following vaccination [3]. For this reason, anecdotal accounts and national media coverage of HPV vaccine side effects, such as unusually high rates of pain at the

() 2009 Elsevier Ltd. All rights reserved

"Corresponding author at: University of North Carolina Gillings School of Global Public Health, Department of Health Behavior and Health Education, 323D Rosenau Hall, CB 7440, Chapel Hill, NC 27599-7440, United States. Tel.: +1 919966 8650; fax: +1 919966 2921. preiter@email.unc.edu (P.L. Reiter)..

Conflict of interest Although we do not believe we have any conflicts of interest, we wish to share the following information in the interest of full disclosure. NTB, the study PI, received a research grant from Merck for a study in 2008-2009 of men's attitudes toward HPV vaccination. NTB has received no honoraria or consulting fees from Merck or GlaxoSmithKline. JSS has received research grants or contracts, honoraria, or consulting fees during the last 4 years from GlaxoSmithKline and Merck. No funds from GlaxoSmithKline or Merck funded these research activities. One co-author from the funding source, the CDC, (SLG) participated in conducting the study and in preparing and submitting this article, though she did not participate in the decision to fund the study. 
injection site and syncope (i.e., fainting), are of serious concern [4-6]. Understanding reported side effects of HPV vaccine is important for fostering widespread vaccine initiation.

In clinical trials of the quadrivalent HPV vaccine, which is currently licensed for clinical use in the US, about $84 \%$ of participants reported experiencing some pain after vaccination, with pain after any one dose ranging from $61 \%$ to $63 \%$ [7]. Additional clinical studies showed similar rates of pain $[8,9]$. Although syncope following vaccination is of particular concern because it is especially common among adolescents and young adults [10,11], syncope after HPV vaccination does not appear to be abnormally high. Data on over 350,000 HPV vaccine doses from Vaccine Safety Datalink (VSD), an active vaccine safety surveillance system in the US, showed no evidence of increased risk of syncope following HPV vaccination, compared to other vaccines administered to adolescents and young adults [12]. Furthermore, almost all cases of syncope reported to the US Vaccine Adverse Event Reporting System (VAERS), a passive surveillance system, have been classified as nonserious [13].

No published studies, to our knowledge, have compared pain reports for HPV vaccine and other vaccines. In this study, we interviewed caregivers of adolescent females about pain and syncope their daughters may have experienced following HPV vaccination and other adolescent vaccines. We also examined whether reported HPV vaccine-related pain was associated with completion of the three-dose vaccination series. We acknowledge that our study relies on parental recall of daughters' vaccination experiences, which may be subject to error. However, we believe our results still provide important insight into how side effects from HPV vaccine compare to those from other adolescent vaccines.

\section{Materials and methods}

\subsection{Study design}

Caregivers of adolescent girls participated in a longitudinal study investigating HPV vaccine decision making, as described in detail by Brewer et al. [14]. In brief, interviewers contacted a probability sample of households in five North Carolina counties using random-digitdialing $(5 \%)$ or a non-overlapping targeted-list frame of directory-listed residential telephone numbers with available recent household demographic information (95\%). They oversampled households likely to contain an adolescent female in the targeted age range of 10-18 years old, households likely to be African American, and rural telephone exchanges [15]. Trained personnel used computer-assisted telephone interviewing equipment to conduct interviews with caregivers, who we refer to as parents for the remainder of this report.

Of 1220 eligible parents contacted, 889 (73\%) completed baseline interviews between July and October 2007 [16]. Of 873 baseline respondents eligible for follow-up, 650 (74\%) completed follow-up interviews between October and November 2008 [14]. This report includes cross-sectional data from parents who reported during the follow-up interview that their daughters had received one or more doses of HPV vaccine $(n=229)$. 
Many of these parents also reported their daughters had received a tetanus booster vaccine $(90 \%, 206 / 229)$ or meningococcal vaccine $(50 \%, 115 / 229)$ at some point prior to the followup interview. Most parents were female (95\%), non-Hispanic white (77\%) or non-Hispanic African American (17\%), married (86\%), and had at least some college education (87\%) (Table 1). The Institutional Review Board at the University of North Carolina approved the study.

\subsection{Measures}

Interviewers asked parents to report the level of pain or discomfort, if any, their daughters experienced from any HPV vaccine doses (1) while in the doctor's office (time of injection) and (2) in the hours and days afterwards. Response options were "no pain or discomfort," "mild," "moderate," "severe pain or discomfort," "daughter didn't say," and "don't know." We selected these responses to be consistent with measures reported in clinical trials of HPV vaccine and other vaccines $[7,17,18]$. When parents reported any pain for the hours and days after injection, interviewers asked how long the pain persisted. Interviewers assessed syncope by asking parents if their daughters experienced a problem with dizziness or fainting following HPV vaccination.

Interviewers assessed pain or discomfort from two other recommended adolescent vaccines, meningococcal vaccine and tetanus booster vaccine (including tetanus and diphtheria [Td] vaccine; and tetanus, diphtheria, and acellular pertussis [Tdap] vaccine), for both the time of injection and in the hours and days afterwards. Response scales were identical to those for HPV vaccine. Tetanus booster (one-dose regimen) and meningococcal (one-dose regimen) vaccines were ideal comparisons since the age recommendations for these vaccines are similar to those for HPV vaccine [19].

Pain levels (no pain, mild, moderate, or severe pain) reported for HPV vaccine at each time period were compared with those reported for tetanus booster and meningococcal vaccines. We created "pain level comparison" variables that had three levels: (1) "same level of pain" reported for HPV vaccine and the comparison vaccine, (2) "less pain from HPV vaccine", and (3) "more pain from HPV vaccine." If a parent responded "daughter didn't say" or "don't know" to either pain question necessary for a comparison, we did not use their data. We also created six dichotomous pain variables (three vaccines and two time periods) with values of 0 for no pain and 1 for any pain (mild, moderate, or severe), not using responses of "daughter didn't say" and "don't know."

Interviewers asked parents to compare the pain or discomfort associated with HPV vaccine to pain or discomfort associated with all other vaccines their daughters had received. Response options were "less pain or discomfort," "about the same," "more pain or discomfort," "does not apply/no shots to compare to," and "don't know."

Parents reported how many doses of HPV vaccine their daughters had received and when they received each one. We defined completion of the HPV vaccine regimen as receipt of all three HPV vaccine doses or receipt of only one or two doses but still on-schedule to complete the regimen within recommended time guidelines, allowing a two month grace 
period (i.e., within 4 months after the first shot or 6 months after the second shot at the time of the follow-up interview) [14].

\subsection{Data analysis}

We calculated frequencies of reported pain or discomfort for all three adolescent vaccines and reported syncope following HPV vaccination. We analyzed the "pain level comparison" variables comparing reported pain from HPV vaccine with that from tetanus booster and meningococcal vaccines using the sign test. We then analyzed the dichotomous pain variables (any pain or no pain) using McNemar's chi-square test to calculate odds ratios (ORs) and confidence intervals (CIs). Binary logistic regression was used to examine the bivariate associations between the two dichotomous HPV vaccine pain variables (at the time of injection and in the hours and days afterwards) and completion of the HPV vaccine regimen. Data were unweighted and analyzed using Intercooled Stata Version 10.1 (College Station, TX). All statistical tests were two-tailed, using a critical alpha of 0.05.

\section{Results}

\subsection{Reported vaccine pain}

The majority of parents $(65 \%, 148 / 229)$ reported their daughters experienced pain or discomfort following receipt of HPV vaccine, with pain more commonly reported at the time of injection $(58 \%, 133 / 229)$ than in the hours and days following vaccination $(45 \%$, 102/229) (Table 2). Few parents reported their daughters experienced moderate or severe pain at the time of injection $(10 \%, 2 \%)$ or in the hours or days following vaccination $(7 \%$, less than $1 \%$ ). Reported pain from HPV vaccine in the hours and days after injection lasted a median of $48 \mathrm{~h}$ (range $=1-336 \mathrm{~h}$ ). No parents reported syncope among their daughters following HPV vaccination, but eight (3\%) reported their daughters felt dizzy or lightheaded.

Most parents reported their daughters experienced some pain or discomfort from tetanus booster vaccine $(84 \%, 174 / 206)$ and meningococcal vaccine $(73 \%, 84 / 115)$. For both vaccines, more parents reported their daughters experienced pain at the time of injection (tetanus booster vaccine $=81 \%$, meningococcal vaccine $=71 \%$ ) than in the hours and days following vaccination (tetanus booster vaccine $=67 \%$, meningococcal vaccine $=46 \%$ ).

\subsection{Reported HPV vaccine pain vs. tetanus and meningococcal vaccine pain}

Among parents whose daughters had received both HPV and tetanus booster vaccines, parents were more likely to report a lower level of pain from HPV vaccine than from tetanus booster vaccine at both the time of injection $(p<0.001)$ and in the hours and days after vaccination $(p<0.001)$ (Fig. 1). Of parents whose daughters had received both HPV and meningococcal vaccines, parents were more likely to report a lower level of pain from HPV vaccine than from meningococcal vaccine at the time of injection $(p=0.003)$ but not in the hours and days following vaccination $(p=1.00)$.

Comparing the dichotomous pain variables for these three adolescent vaccines yielded a similar pattern of findings. More parents reported their daughters experienced pain from 
tetanus booster vaccine at the time of injection $(83 \%, 154 / 186)$ than from HPV vaccine $(62 \%, 116 / 186)(\mathrm{OR}=6.43,95 \% \mathrm{CI}: 2.88-16.90, p<0.001)$ among those whose daughters had received both vaccines. A higher proportion of parents also reported their daughters experienced pain from tetanus booster vaccine $(70 \%, 133 / 191)$ than from HPV vaccine $(45 \%, 85 / 191)(\mathrm{OR}=4.43,95 \% \mathrm{CI}: 2.45-8.57, p<0.001)$ in the hours and days following vaccination. Among parents whose daughters had received both HPV and meningococcal vaccines, more reported their daughters experienced pain from meningococcal vaccine at the time of injection $(74 \%, 77 / 104)$ than from HPV vaccine $(59 \%, 61 / 104)(\mathrm{OR}=3.29,95 \% \mathrm{CI}$ : $1.36-9.07, p=0.005)$. No difference was found for reported pain in the hours and days following meningococcal and HPV injections (49\%, 50/103 vs. 46\%, 47/103) (OR = 1.20, $95 \%$ CI: $0.57-2.56, p=0.728)$. Sensitivity analyses stratifying by daughter's age and number of HPV vaccine doses received confirmed the robustness of our findings (data not shown).

\subsection{Reported HPV vaccine pain vs. all other vaccine pain}

Most parents said HPV vaccine caused their daughters the same amount of pain or discomfort at the time of injection compared to all other vaccines received (69\%, 159/229). Some parents said their daughters experienced less pain from HPV vaccine compared to all other vaccines at the time of injection $(17 \%, 38 / 229)$, while even fewer said it caused more pain $(12 \%, 27 / 229)$. Reports of pain or discomfort in the hours and days after vaccination followed the same pattern (same pain: 62\%, 142/229; less pain: 25\%, 58/229; and more pain: $11 \%, 25 / 229)$.

\subsection{Influence of HPV vaccine pain on vaccine regimen completion}

Reported pain from HPV vaccination did not influence timely uptake of subsequent doses of HPV vaccine. Parents of daughters who had completed the three dose series (or were within recommended time guidelines to do so) reported pain or discomfort at the time of injection nearly as often as parents whose daughters were late for their second or third dose $(62 \%$, $114 / 185$ vs. $68 \%, 19 / 28)(\mathrm{OR}=0.76,95 \%$ CI: $0.33-1.77, p=0.53)$. Similarly, reported pain or discomfort in the hours and days after vaccination was equally common among parents whose daughters were within and not within recommended time guidelines for completing the vaccine regimen $(47 \%, 89 / 188$ vs. $43 \%, 13 / 30)(\mathrm{OR}=1.18,95 \%$ CI: $0.54-2.56, p=$ $0.68)$.

\section{Discussion}

\subsection{Main findings}

Anecdotal accounts and national media coverage have suggested that severe pain at the injection site and syncope are particularly common adverse events following HPV vaccination [4-6]. While many parents in our study reported their daughters experienced pain or discomfort after receiving HPV vaccine, the reported pain was less frequent and less severe than that associated with other adolescent vaccines and did not affect completion of the HPV vaccine regimen. To our knowledge, this study is the first to examine HPV vaccine-related pain outside the context of a clinical vaccine efficacy trial and the first to compare reported pain from HPV vaccine with pain from other adolescent vaccines. 
A majority of parents reported their daughters experienced pain or discomfort after receiving HPV vaccine, as well as after receiving meningococcal or tetanus booster vaccines.

Reported pain in our community-based study was similar to previous clinical trials involving these vaccines (HPV vaccine: 73-84\% [7-9]; tetanus booster vaccine: $64-87 \%$ [17,20,21]; meningococcal conjugate vaccine: $69 \%$ [18]).

Of greater interest, most parents indicated their daughters experienced the same amount of pain or less pain from HPV vaccine than with tetanus booster and meningococcal vaccines. Comparatively few parents indicated their daughter experienced more pain from HPV vaccine. Similar results were obtained when parents were asked to compare HPV vaccine to all other vaccines their daughters had received. Reported pain from HPV vaccine also did not appear to influence completion of the HPV vaccine regimen, which is consistent with the findings of HPV vaccine clinical trials in which few participants discontinued the vaccine regimen due to adverse reactions [7].

Few parents reported their daughters experienced dizziness after receiving HPV vaccine and none reported syncope, which is reassuring given the concerns about syncope after HPV vaccine receipt. These findings support both results from HPV vaccine clinical trials (4\% reported dizziness) [7] and evidence suggesting reports of serious occurrences of syncope are not common following HPV vaccination [13,22]. However, we acknowledge that a much larger sample size would be required to assess increased risk of syncope following HPV vaccination adequately.

\subsection{Strengths and limitations}

Our study's strengths include assessment of a sizable sample of parents with daughters in the recommended age range for HPV vaccine and other adolescent vaccines, a good response rate, and examination of pain at both the time of injection and in the hours and days after vaccination.

As previously mentioned, our study's main limitation was reliance on parental recall of daughters' vaccination experiences. However, we believe the pain comparisons were valid because the reported pain levels for each of the three vaccines were comparable to previous studies, several of which used the same categories in measuring pain level $[7,17,18]$. We did not collect vaccination dates for tetanus booster and meningococcal vaccines, so we do not know when they were received in relation to HPV vaccine. We were not able to examine dose-specific reports of pain, as all HPV vaccine pain items referred to any doses received so far. Clinical trials have, however, showed that reported pain was almost equivalent across the threes doses [7]. Our study focused on common adolescent vaccines, but findings could differ if HPV vaccine were compared to other vaccines with lower levels of reactogenicity. As our study included parents from one area of North Carolina who had a telephone and spoke English, the generalizability of the findings is not yet known.

\subsection{Conclusions}

We believe our results have important public health implications. Future programs designed to increase initiation and completion of the HPV vaccine regimen may inform parents, daughters, and healthcare providers that pain from HPV vaccination has been reported by 
parents as being similar to or less than pain from other adolescent vaccines their daughters have received. Such information can help alleviate concerns and fears regarding adverse events following HPV vaccination not only among parents but also healthcare providers, as both groups have expressed concern over possible side effects from the vaccine [3,23-25]. An important next step is to prospectively assess adolescent girls' pain reports from HPV and other vaccines and associated vaccine regimen completion to confirm our findings.

\section{Acknowledgments}

This study was funded by grants from the Centers for Disease Control and Prevention (S3715-25/25), the American Cancer Society (MSRG-06-259-01-CPPB), and the National Cancer Institute (R25 CA57726).

\section{References}

[1]. Centers for Disease Control and Prevention (CDC). Vaccination coverage among adolescents aged 13-17 years-United States, 2007. MMWR Morb Mortal Wkly Rep. 2008; 57(40):1100-3. [PubMed: 18846032]

[2]. Grant, D.; Kravitz-Wirtz, N.; Breen, N.; Tiro, JA.; Tsui, J. One in four California adolescent girls have had human papillomavirus vaccination. UCLA Center for Health Policy Research; Los Angeles, CA: 2009.

[3]. Reiter PL, Brewer NT, Gottlieb SL, McRee AL. Smith JS. Parents' health beliefs and HPV vaccination of their adolescent daughters. Soc Sci Med. 2009; 69(3):475-80. [PubMed: 19540642]

[4]. Associated Press. Cervical cancer vaccine called most painful shot. Fox News [Internet]. Jan 4. 2008 Available from: http://www.foxnews.com/story/0,2933,320132,00.html

[5]. Associated Press. Some girls fainting after receiving HPV vaccine. MSNBC [Internet]. Jan 3. 2008 Available from: http://www.msnbc.msn.com/id/22492557/

[6]. Kotz, D. 5 things to consider before getting the HPV vaccine. U.S. News and World Report [Internet]. Sep 2. 2008 Available from: http://health.usnews.com/articles/health/cancer/ 2008/09/02/5-things-to-consider-before-getting-the-hpv-vaccine.html

[7]. Food and Drug Administration. Merck \& Co. Whitehouse Station, NJ: Product approval information-licensing action [package insert]. gardasil (quadrivalent human papillomavirus types 6, 11, 16, 18). Available from: http://www.fda.gov/cber/label/HPVmer060806LB.pdf

[8]. Kang S, Kim KH, Kim YT, Kim YT, Kim JH, Song YS, et al. Safety and immunogenicity of a vaccine targeting human papillomavirus types $6,11,16$ and 18: a randomised, placebo-controlled trial in 176 Korean subjects. Int J Gynecol Cancer. 2008; 18(5):1013-9. [PubMed: 17986242]

[9]. Perez G, Lazcano-Ponce E, Hernandez-Avila M, Garcia PJ, Munoz N, Villa LL, et al. Safety, immunogenicity, and efficacy of quadrivalent human papillomavirus (types $6,11,16,18$ ) L1 virus-like-particle vaccine in Latin American women. Int J Cancer. 2008; 122(6):1311-8. [PubMed: 18000825]

[10]. Kroger AT, Atkinson WL, Marcuse EK, Pickering LK, Advisory Committee on Immunization Practices (ACIP) Centers for Disease Control and Prevention (CDC). General recommendations on immunization: recommendations of the Advisory Committee on Immunization Practices (ACIP). MMWR Recomm Rep. 2006; 55(RR-15):1-48. [PubMed: 17136024]

[11]. Centers for Disease Control Prevention (CDC). Syncope after vaccination-United States, January 2005-July 2007. MMWR Morb Mortal Wkly Rep. 2008; 57(17):457-60. [PubMed: 18451756]

[12]. Gee, J.; Naleway, A.; Shui, I. Vaccine Safety Datalink Project: monitoring the safety of quadrivalent human papillomavirus vaccine (HPV4). Presented at: Advisory Committee on Immunization Practices Meeting; October 22, 2008;

[13]. Slade BA, Leidel L, Vellozzi C, Woo EJ, Hua W, Sutherland A, et al. Postlicensure safety surveillance for quadrivalent human papillomavirus recombinant vaccine. JAMA. 2009; 302(7): 750-7. [PubMed: 19690307] 
[14]. Brewer, NT.; Gottlieb, SL.; Reiter, PL.; McRee, AL.; Liddon, N.; Markowitz, LE., et al. Working Paper. 2009. Longitudinal predictors of HPV vaccine uptake.

[15]. U.S. Census Bureau. Census glossary. 2008. UpdatedAvailable from: http:// factfinder.census.gov/home/en/epss/glossary_a.html

[16]. Hughes J, Cates JR, Liddon N, Smith JS, Gottlieb SL, Brewer NT. Disparities in how parents are learning about the human papillomavirus vaccine. Cancer Epidemiol Biomarkers Prev. 2009; 18(2):363-72. [PubMed: 19190161]

[17]. Halperin SA, Sweet L, Baxendale D, Neatby A, Rykers P, Smith B, et al. How soon after a prior tetanus-diphtheria vaccination can one give adult formulation tetanus-diphtheria-acellular pertussis vaccine? Pediatr Infect Dis J. 2006; 25(3):195-200. [PubMed: 16511379]

[18]. Keyserling H, Papa T, Koranyi K, Ryall R, Bassily E, Bybel MJ, et al. Safety, immunogenicity, and immune memory of a novel meningococcal (groups A, C, Y, and W-135) polysaccharide diphtheria toxoid conjugate vaccine (MCV-4) in healthy adolescents. Arch Pediatr Adolesc Med. 2005; 159(10):907-13. [PubMed: 16203934]

[19]. Centers for Disease Control and Prevention. Recommended immunization schedules for persons aged 0-18 years-United States, 2008. MMWR Morb Mortal Wkly Rep. 2007; 56(51\&52):Q14.

[20]. Pichichero ME, Blatter MM, Kennedy WA, Hedrick J, Descamps D. Friedland LR. Acellular pertussis vaccine booster combined with diphtheria and tetanus toxoids for adolescents. Pediatrics. 2006; 117(4):1084-93. [PubMed: 16585302]

[21]. Zepp F, Knuf M, Habermehl P, Mannhardt-Laakmann W, Howe B, Friedland LR. Safety of reduced-antigen-content tetanus-diphtheria-acellular pertussis vaccine in adolescents as a sixth consecutive dose of acellular pertussis-containing vaccine. J Pediatr. 2006; 149(5):603-10. [PubMed: 17095328]

[22]. Calugar, A. Quadrivalent human papillomavirus vaccine (HPV4): post-licensure safety update, Vaccine Adverse Event Reporting System (VAERS), United States. Presented at: Advisory Committee on Immunization Practices Meeting; October 22, 2008;

[23]. Brewer NT, Fazekas KI. Predictors of HPV vaccine acceptability: a theory-informed, systematic review. Prev Med. 2007; 45(2-3):107-14. [PubMed: 17628649]

[24]. Ishibashi KL, Koopmans J, Curlin FA, Alexander KA, Ross LF. Paediatricians' attitudes and practices towards HPV vaccination. Acta Paediatr. 2008; 97(11):1550-6. [PubMed: 18671696]

[25]. Esposito S, Bosis S, Pelucchi C, Begliatti E, Rognoni A, Bellasio M, et al. Pediatrician knowledge and attitudes regarding human papillomavirus disease and its prevention. Vaccine. 2007; 25(35):6437-46. [PubMed: 17673339] 


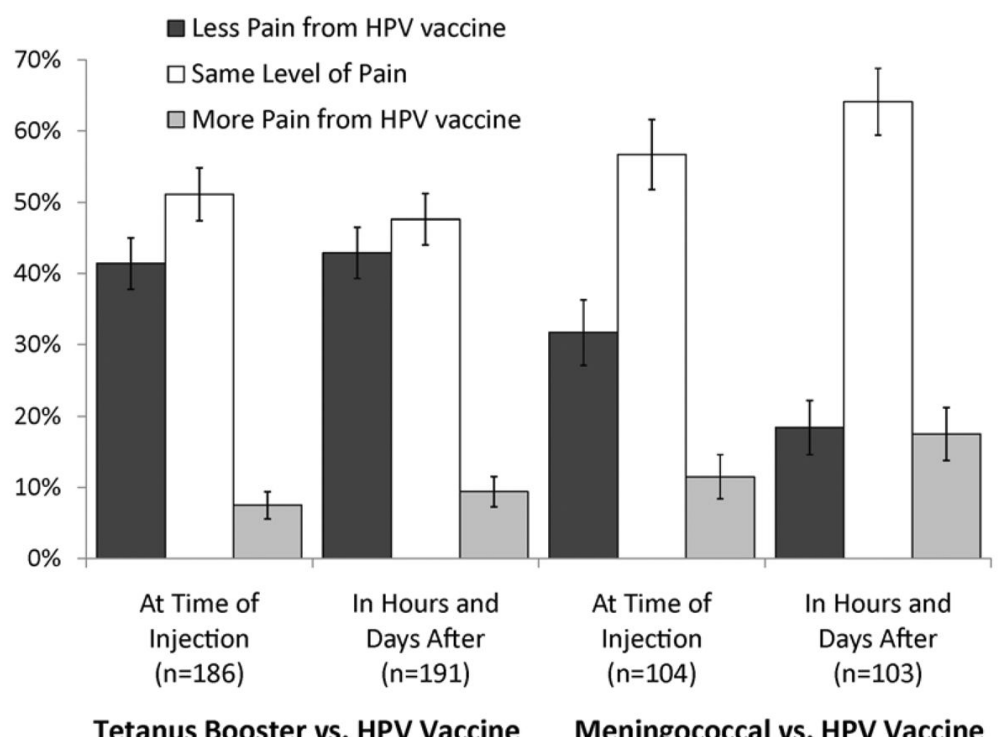

Fig. 1.

Parents' reports of daughters' pain level from HPV vaccine compared to pain levels from tetanus booster and meningococcal vaccines. Note: Bars indicate the standard errors of the proportions. Pain levels (no pain, mild, moderate, or severe pain) reported for HPV vaccine were compared with those reported for tetanus booster and meningococcal vaccines in creating the three categories of "less pain from HPV vaccine," "same level of pain," and "more pain from HPV vaccine." HPV: human papillomavirus. 


\section{Table 1}

Characteristics of parents and their daughters who had received at least one dose of human papillomavirus (HPV) vaccine $(n=229)$.

\begin{tabular}{|c|c|c|}
\hline & Mean (SD) & \\
\hline Daughter characteristics Age (years) & $16.4(2.3)$ & \\
\hline \multirow[t]{2}{*}{ Parent characteristics Age (years) } & $45.2(6.4)$ & \\
\hline & & $\mathrm{n}(\%)$ \\
\hline \multicolumn{3}{|l|}{ Sex } \\
\hline \multicolumn{2}{|l|}{ Female } & $218(95)$ \\
\hline \multicolumn{2}{|l|}{ Male } & $11(5)$ \\
\hline \multicolumn{3}{|l|}{ Race/ethnicity } \\
\hline \multicolumn{2}{|l|}{ White, non-Hispanic } & $177(77)$ \\
\hline \multicolumn{2}{|l|}{ African American, non-Hispanic } & $39(17)$ \\
\hline \multicolumn{2}{|l|}{ Other } & $13(6)$ \\
\hline \multicolumn{3}{|l|}{ Marital status } \\
\hline \multicolumn{2}{|l|}{ Married/living as married } & $198(86)$ \\
\hline \multicolumn{2}{|c|}{ Other (divorced, widowed, separated, never married) } & $31(14)$ \\
\hline \multicolumn{3}{|l|}{ Education level } \\
\hline \multicolumn{2}{|l|}{ Some college or more } & $200(87)$ \\
\hline \multicolumn{2}{|l|}{ High school or less } & $29(13)$ \\
\hline \multicolumn{3}{|l|}{ Annual household income } \\
\hline \multicolumn{2}{|l|}{ Less than $\$ 60,000$} & $82(36)$ \\
\hline \multicolumn{2}{|l|}{$\$ 60,000$ and over } & $136(59)$ \\
\hline \multicolumn{2}{|l|}{ Missing } & $11(5)$ \\
\hline \multicolumn{3}{|l|}{ Residence type } \\
\hline \multicolumn{2}{|l|}{ Urban } & $108(47)$ \\
\hline \multicolumn{2}{|l|}{ Rural } & $121(53)$ \\
\hline
\end{tabular}

Note. SD: standard deviation. 
Table 2

Parents' reports of daughters' pain or discomfort from HPV, tetanus booster, and meningococcal vaccines.

\begin{tabular}{|c|c|c|c|c|c|c|}
\hline & None $n(\%)$ & Mild $n(\%)$ & Moderate $n(\%)$ & Severe $n(\%)$ & $\begin{array}{r}\text { Daughter } \\
\text { didn't say } n \\
(\%)\end{array}$ & Don't know n(\%) \\
\hline $\begin{array}{l}\text { Pain from HPV vaccine at time of } \\
\text { injection }\end{array}$ & $80(35)$ & $104(45)$ & $24(10)$ & $5(2)$ & $13(6)$ & $3(1)$ \\
\hline $\begin{array}{l}\text { Pain from tetanus booster vaccine } \\
\text { at time of injection }\end{array}$ & $35(17)$ & $102(50)$ & $58(28)$ & $6(3)$ & $1(<1)$ & $4(2)$ \\
\hline $\begin{array}{l}\text { Pain from meningococcal vaccine } \\
\text { at time of injection }\end{array}$ & $28(24)$ & $66(57)$ & $16(14)$ & $0(0)$ & $2(2)$ & $3(3)$ \\
\hline $\begin{array}{l}\text { Pain from HPV vaccine in hours } \\
\text { and days after }\end{array}$ & $116(51)$ & $85(37)$ & $16(7)$ & $1(<1)$ & $7(3)$ & $4(2)$ \\
\hline $\begin{array}{l}\text { Pain from tetanus booster vaccine } \\
\text { in hours and days after }\end{array}$ & $62(30)$ & $92(45)$ & $42(20)$ & $4(2)$ & $3(1)$ & $3(1)$ \\
\hline $\begin{array}{l}\text { Pain from meningococcal vaccine } \\
\text { in hours and days after }\end{array}$ & $56(49)$ & $48(42)$ & $5(4)$ & $0(0)$ & $4(3)$ & $2(2)$ \\
\hline
\end{tabular}

Note. Row percents may not sum to $100 \%$ due to rounding. HPV: human papillomavirus. 